\title{
Molecular dissection of intra-tumoral heterogeneity in human epithelial carcinoma
}

\author{
Naisana S Asli* \\ Discipline of Life Sciences, Faculty of Dentistry, University of Sydney, Westmead Hospital, Westmead, NSW 2145, Australia
}

As a major component of differential response to therapy, tumor heterogeneity has attracted significant clinical attention. It adds contextual attributes to the emerging so-called personalized therapy, and can retrospectively illuminate on the fundamental concepts in cancer biology like origin and horizontal evolution of cancer. Tumor heterogeneity manifests at several levels; Inter-tumoral heterogeneity refers to variabilities between similar histological tumor subtypes in different patients, same tumors at different stages of cancer progression (temporal variation) or separate masses or macrometastases of same tumor type at different locations (spatial variation). Epithelial carcinoma of the ovaries has served as a classic example for intertumoral heterogeneity with at least 5 distinct histological subtypes, all with different developmental origins, chemotherapy response profiles, and growth dynamics [1]. Genetic heterogeneity between tumor subtypes have been studied for few decades but advances in genomics and sequencing have added more detailed functional aspects to both driver and passenger mutations occurring in individual tumors. With respect to ovarian epithelial carcinoma, high-through-put sequencing has revealed several levels of genetic and epigenetic inter-tumoral heterogeneity translating into discordant clinical behaviors [2-5]. Evidence support a self-perpetuating nature in genetic heterogeneity of tumors that complicate clinical outcomes and ultimately result in poor prognosis. At the level of individual tumors, there is as well evidence for spatial and temporal intra-tumoral heterogeneity reflecting heterogeneous cellular compartments. Intra-tumoral heterogeneity can manifest at multiple functional levels including morphological variations or changes to intracellular processes. Phenotypic heterogeneity in tumors was described in early years of cancer biology with more in depth revisits in recent years defining functional phenotypes along the epithelial and mesenchymal axes. Epithelial mesenchymal transition (EMT) is a central process during embryogenesis defined as plastic changes between epithelial and mesenchymal morphologies with downstream outcomes such as cellular motility and potency. In tumors, EMT has received its first recognistion as a core process during metastasis [6]. Later studies have however defined a more fundamental role for EMT in the so-called cancer stem cell or tumor initiating compartment within the tumors that are essentially the cellular drivers of tumor mass [7-10]. We have been using human epithelial carcinoma as a model system to dissect functional pathways underlying cellular heterogeneity within tumors. We have observed distinct epithelial and mesenchymal compartments in a variety of epithelial carcinoma cell lines including COLO316 epithelial ovarian carcinoma cells (Figure 1). Our findings as well suggest discrete migration profiles and growth dynamics for each subgroup, with a large degree of morphological plasticity. Our findings so far, suggest for cells with epithelial morphologies to retain higher growth dynamics but less migration capacity with reverse phenotypes observed for mesenchymal cells (data not shown). Cells within individual tumors can as well demonstrate asynchronous cell cycle profiles not only at the level of cell cycle entry time but in the overall length of the cell cycle and individual phases [11]. In pluripotent stem cells, cell cycle events and cell fate decisions are tightly orchestrated, resulting in distinct cycle phase-associated differentiation outcomes, and differential levels of response to microenvironmental stimuli [12]. Our analysis of cell cycle on bulk cultures of COLO316, using a double RNA and DNA staining method described before [13] showed a heterogeneous distribution pattern of cells between four discrete phases (G0, G1, S and G2/M) with a large fraction of the cells (>16\%) in the quiescent or G0 phase of the cell cycle. We have as well observed a high level of Carboplatin resistance/evasion in these cells in alignment with the high number of quiescent cells which support the notion that the quiescent cellular compartment might act at the core of chemoevasion/-resistance. Functional heterogeneity within a tumor can result from spatial topography of the tumor mass and the geographical coordinates of cellular compartments. As the tumor grows, distance from blood vessels creates a spectrum of accessible oxygen, causing different levels of hypoxia for individual cells/clones within a tumor. This phenomenon can result in spatially distinct metabolic signatures as demonstrated during the progression of breast cancer [14]. Metabolic heterogeneity, can also shape downstream of spatial nutrient/metabolite compartments in the tumor microenvironment as observed in human non-small cell lung cancer [15]. Our own findings using COLO316 ovarian epithelial carcinoma cell lines as well suggest a large degree of metabolic heterogeneity, evidenced by different uptakes of Mitotracker Green FM as a read-out for metabolic activity (Figure 1C). Given the functional role of metabolic profiles during tumorigenesis, this observation suggests possible downstream oncogenic heterogeneity as a direct outcome of differential metabolic signatures.

Tumor heterogeneity is increasingly evident in genetic and cellular landscapes. Although the possible causative relationship between these is yet to be delineated, its complexity implies that tumors should be viewed as constantly evolving dynamic entities with temporal variability in sub-clone compositions. In fact, as tumors grow, continuous change in the tumor microenvironment resulting from changes in the tumor or stromal cellular context, the local secretomes and/or circulating factors, build a selective pressure that greatly contributes to tumor evolution.

Correspondence to: Dr. Naisana S Asli, Lecturer, Life Sciences, Faculty of Dentistry, University of Sydney, Westmead NSW 2145, Australia, Tel: 02 88904818; E-mail: naisana.seyedasli@sydney.edu.au

Received: September 14, 2016; Accepted: September 27, 2016; Published: September 29, 2016 
A
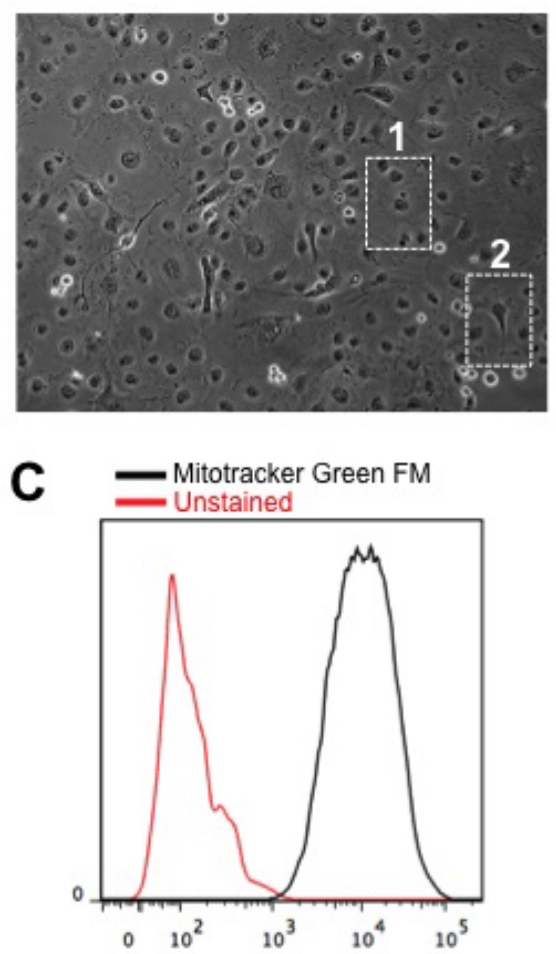

B

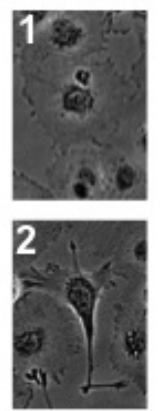

D
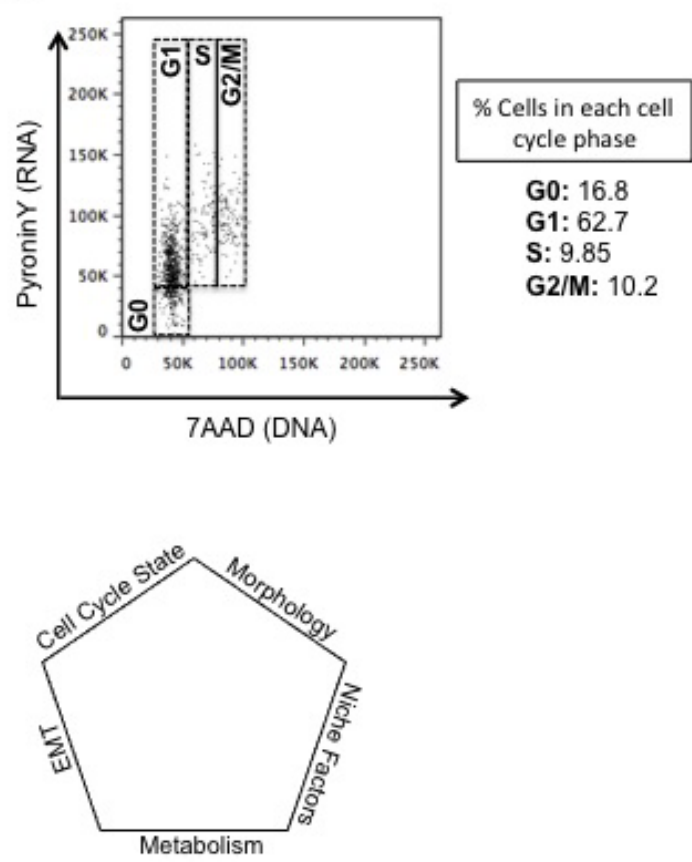

Figure 1. Different levels of cellular heterogeneity in human epithelial ovarian carcinoma cells. (A) Phenotypic heterogeneity in COLO316 cells. Note discrete mesenchymal and epithelial morphologies in the bulk cultures. (B) Cell cycle asynchrony in COLO316 cells. PyroninY levels depict bulk RNA synthesis as a read-out for metabolic activity. 7AAD stains for DNA to differentiate between 2N and 4N DNA content. (C) Metabolic heterogeneity in COLO316 cells. Mitotracker Green FM stains the mitochondria as a read-out for metabolic activity. (D) Illustration of cellular heterogeneity as a multifactorial event.

Learning about this process will favor better targeted therapy and more promising clinical outcomes.

\section{Acknowledgements}

The ovarian carcinoma project has greatly benefitted from the intellectual support of Professor Anna deFazio and her team at the Westmead Institute of Medical Research that needs to be acknowledged.

\section{References}

1. McCluggage WG (2011) Morphological subtypes of ovarian carcinoma: a review with emphasis on new developments and pathogenesis. Pathology 43: 420-432. [Crossref]

2. Bai H (2016) Genetic and epigenetic heterogeneity of epithelial ovarian cancer and the clinical implications for molecular targeted therapy. J Cell Mol Med 20: 581-93.

3. Blagden SP (2015) Harnessing Pandemonium: The Clinical Implications of Tumor Heterogeneity in Ovarian Cancer. Front Oncol 5: 149. [Crossref]

4. Lee JY (2015) Tumor evolution and intratumor heterogeneity of an epithelial ovarian cancer investigated using next-generation sequencing. BMC Cancer 15: 85 .

5. Patch AM, Christie EL, Etemadmoghadam D, Garsed DW, George J, et al. (2015) Whole-genome characterization of chemoresistant ovarian cancer. Nature 521: 489494. [Crossref]

6. Heerboth S, Housman G, Leary M, Longacre M, Byler S, et al. (2015) EMT and tumor metastasis. Clin Transl Med 4: 6. [Crossref]

7. Guo W, Keckesova Z, Donaher JL, Shibue T, Tischler V, et al. (2012) Slug and Sox 9 cooperatively determine the mammary stem cell state. Cell 148: 1015-1028. [Crossref]

8. Mani SA, Guo W, Liao MJ, Eaton EN, Ayyanan A, et al. (2008) The epithelialmesenchymal transition generates cells with properties of stem cells. Cell 133: 704715. [Crossref]

9. Ye X, Tam WL, Shibue T (2015) Distinct EMT programs control normal mammary stem cells and tumour-initiating cells. Nature 525: 256-260. [Crossref]

10. Asli NS, Harvey RP (2013) Epithelial to mesenchymal transition as a portal to stem cell characters embedded in gene networks. Bioessays 35: 191-200. [Crossref]

11. Haass NK, Beaumont KA, Hill DS, Anfosso A, Mrass P, et al. (2014) Real-time cell cycle imaging during melanoma growth, invasion, and drug response. Pigment Cell Melanoma Res 27: 764-776. [Crossref]

12. Pauklin S, Vallier L (2013) The cell-cycle state of stem cells determines cell fate propensity. Cell 155: 135-147. [Crossref]

13. Xin L, Lawson DA, Witte ON (2005) The Sca-1 cell surface marker enriches for prostate-regenerating cell subpopulation that can initiate prostate tumorigenesis. Proc Natl Acad Sci 102: 6942-6947.

14. Jiang L (2012) Localized hypoxia results in spatially heterogeneous metabolic signatures in breast tumor models. Neoplasia 4: 732-41.

15. Hensley CT, Faubert B, Yuan Q, Lev-Cohain N, Jin E, et al. (2016) Metabolic Heterogeneity in Human Lung Tumors. Cell 164: 681-694. [Crossref]

Copyright: (C2016 Asli NS. This is an open-access article distributed under the terms of the Creative Commons Attribution License, which permits unrestricted use, distribution, and reproduction in any medium, provided the original author and source are credited. 